\title{
ACOMPAÑANDO A LAS MUJERES GESTANTES VÍCTIMAS DE VIOLENCIA DE GÉNERO. ASPECTOS ÉTICOS PARA LAS MATRONAS
}

Júlia Martín Badia ${ }^{1}$

Correo electrónico: juliamartin.correu@gmail.com

1. Doctora en Filosofía, Facultad de Filosofía, Universidad de Barcelona, Barcelona.

Recibido: 24/07/2019 Aceptado: 08/11/2019

(c) (i) () 


\section{RESUMEN}

El objetivo del artículo es definir un marco ético dónde se vertebren los elementos esenciales (no necesariamente los únicos) que las matronas deben tener en cuenta cuando atienden a mujeres embarazadas que están sufriendo violencia de género (VG). La propuesta girará en torno al concepto de acompañamiento, entendido como el proceso por el cual se da apoyo a una persona vulnerable para ayudarla a ganar cuotas de autonomía. Se sugerirá un modelo de acompañamiento biopsicosocial de las mujeres gestantes y víctimas de violencia con tres dimensiones: en primer lugar, el acompañamiento es un proceso a través del cual la matrona debe ayudar a la mujer a empoderarse a sí misma; en segundo lugar, es un proceso en el cual la matrona debe construir un entorno de confianza y respeto en la consulta; y, en tercer lugar, requiere unas adecuadas relaciones interprofesionales. El artículo se centrará en las dos primeras dimensiones, que son dos caras de la misma moneda, y se referirá solo brevemente a la tercera, puesto que va más allá del ámbito de las matronas y, por tanto, del propósito del presente trabajo. El método utilizado ha sido el análisis y la reflexión filosófico-racional.

Palabras clave: Ética; violencia de género; matronas; acompañamiento; autonomía; reconocimiento. 


\begin{abstract}
The aim of this paper is to define an ethical framework that intertwines the essentialbut not the only-elements that midwives should take into account when assisting pregnant women who suffer from gender violence $(\mathrm{GV})$. Our proposal will focus on the idea of accompaniment, which can be defined as the process through which support is given to a vulnerable person in order to help her/him gain autonomy. A model of a biopsychosocial accompaniment of pregnant women suffering from gender violence will be suggested. It has three dimensions: firstly, it is a process through which midwives help women empower themselves; secondly, it is a process through which midwives build up an environment of trust and respect in the medical consultation; and thirdly, it requires an appropriate teamwork. This paper will focus on the first two dimensions, which are the two sides of the same coin, and will only briefly address the third one, as it goes far beyond the field of midwifery and thus beyond the aim of this article. The methodology used to develop this paper is the philosophical-rational analysis and reflection.
\end{abstract}

Keywords: Ethics; Gender Violence; Midwives; Accompaniment; Autonomy; Recognition. 


\section{INTRODUCCIÓN: LA NECESIDAD DE ACOMPAÑAMIENTO}

Todas las mujeres están en riesgo de sufrir violencia de género (VG). No hay un perfil que se pueda establecer en función de factores como la edad, la clase social, la religión, la etnia, la cultura, la educación, la orientación sexual o la aptitud física o mental (Velasco, 2008; Estévez, 2017; entre otros). Que una mujer sufra VG dependerá del marco mental del agresor que, a su vez, responde al marco mental de la sociedad, todavía patriarcal.

El acompañamiento es el proceso por el cual se da apoyo a una persona vulnerable para ayudarla a ganar cuotas de autonomía. Debe ser un proceso biopsicosocial, porque debe atender todas las dimensiones de la persona y su proyecto vital.

El embarazo es un momento de gran vulnerabilidad, porque el cuerpo sufre cambios físicos y hormonales que la mujer debe gestionar y porque implica tener que empezar a cuidar de un ser que todavía es más vulnerable que ella, lo cual puede acarrearle dudas y miedos. Es por este motivo que el acompañamiento que hacen las matronas debe consistir en ayudar a las mujeres gestantes a desarrollar su autonomía, fortaleciendo sus capacidades de autocuidado y de cuidado de otros, siempre teniendo en cuenta el cuidado físico, emocional y social, tanto de la madre como del bebé.

Sin embargo, el acompañamiento no debe acabar aquí. Precisamente porque no hay un perfil de víctima de VG, las matronas deben estar preparadas para detectar señales de que una mujer está sufriendo, o está en riesgo de sufrir, violencia (física, psicológica o aislamiento social), y debe tener herramientas para ayudarla a no perder o a recuperar su proyecto vital, más allá de la maternidad y de su relación de pareja.

El acompañamiento biopsicosocial, entendido como el fortalecimiento de las capacidades de cuidado y la detección de indicadores de riesgo, debe hacerse no sólo ante una sospecha de VG, sino para cualquier mujer embarazada, desde la primera visita y durante todo el proceso asistencial, incluyendo el postparto. 
El presente artículo tiene por objetivo ahondar en el acompañamiento a las mujeres embarazadas y víctimas de violencia, proponiendo un modelo que es aplicable no solo ante la evidencia de violencia, sino incluso antes de que aparezca la sospecha, para facilitar la detección de indicadores de riesgo. El modelo de acompañamiento propuesto tiene tres dimensiones: en primer lugar, es un proceso en el que la matrona debe ayudar a la mujer a empoderarse a sí misma; en segundo lugar, es un proceso en el que la matrona debe construir un entorno de confianza y respeto en la consulta; y en tercer lugar, requiere unas relaciones interprofesionales adecuadas. Esta tercera dimensión se comentará brevemente, dado que se aleja del ámbito de las matronas.

El método usado es el análisis y la reflexión filosófico-racional.

\section{ACOMPAÑAMIENTO COMO PROCESO DE EMPODERAMIENTO DE LA MUJER GESTANTE Y VÍCTIMA DE VIOLENCIA DE GÉNERO}

El embarazo y el parto constituyen un proceso de gran vulnerabilidad: hay cambios físicos y hormonales; surge la responsabilidad para con otro ser vivo; y en el momento de dar a luz se depende de otras personas (matronas, ginecólogos...), a las que se les muestra la propia intimidad. En este proceso, las mujeres pueden ver vulnerados sus derechos reproductivos y su integridad física y moral, como ocurre en las situaciones de violencia obstétrica.

Si, además, hay una situación de VG, la vulnerabilidad aumenta, en la medida en que la violencia disminuye la autoestima y genera dependencia respecto del maltratador. Esto no quiere decir que, por ser víctima de VG, la mujer no pueda tener autonomía. La autonomía se define, en sentido filosófico, como el derecho a tomar decisiones y la capacidad de ejercer este derecho. La capacidad puede estar disminuida a consecuencia de la violencia, pero el derecho se mantiene siempre.

El acompañamiento debe basarse, por lo tanto, en ayudar a la mujer a recuperar su autonomía como capacidad de tomar decisiones. La matrona debe acompañar a la mujer en el proceso de empoderarse a sí misma en la toma de decisiones. Entendido de este modo, el acompañamiento tiene tres pilares (fig. 1): 


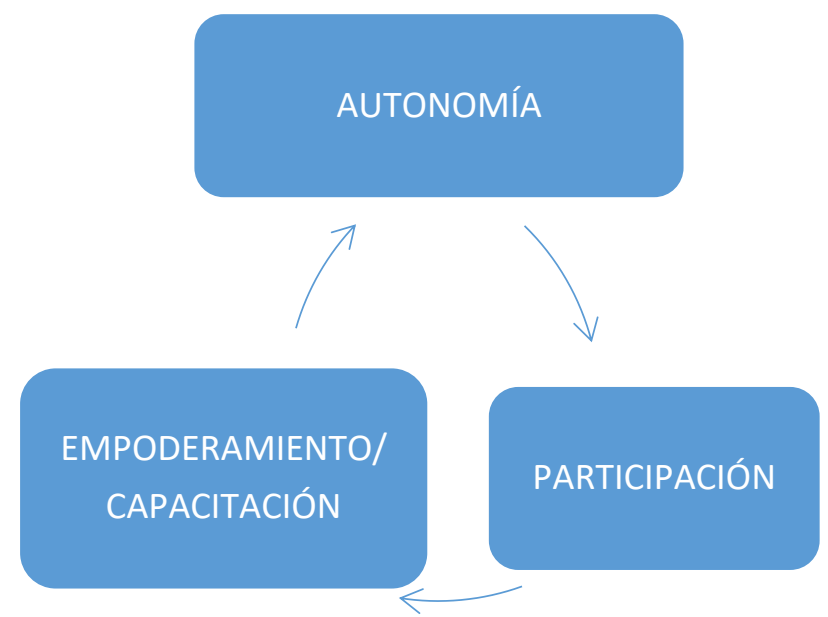

Figura 1. Modelo de acompañamiento como proceso de empoderamiento de la mujer gestante y víctima de violencia de género (elaboración propia).

El esquema anterior es válido para multitud de situaciones, dentro y fuera del ámbito clínico. Veamos cómo las matronas pueden aplicarlo a la situación de las mujeres gestantes y víctimas de VG.

El objetivo del proceso de apoderamiento de la mujer embarazada es la adquisición de autonomía para el cuidado de ella misma y de su bebé. Si la mujer está siendo víctima de VG, la matrona deberá procurar que la mujer pueda ejercer su derecho a tomar decisiones libres, esto es, no coaccionadas por su pareja, en relación con ese cuidado.

Además, la matrona deberá ayudar a la mujer a gestionar la situación de violencia. Es un proceso a largo plazo: la mujer debe aceptar que está siendo víctima de violencia, tomar conciencia de sus emociones, decidir separarse, preparar el plan de fuga, etc. Mientras tanto, ejercer su derecho a decidir a corto plazo en relación con ella misma como persona (autocuidado psíquico y social), con su salud física en general, y con su embarazo y parto en particular, puede ayudarla a fortalecer su capacidad de decisión para, más adelante, tomar decisiones en relación a su situación de violencia.

Por lo tanto, el objetivo es que la mujer recupere el ejercicio de su derecho a decidir en todas las áreas de su vida, empezando por cómo reconstruir su identidad, y cómo cuidar de su salud y de la de su bebé, y terminando por cómo gestionar su relación de pareja.

El modo de conseguir este objetivo es fortalecer las capacidades de la mujer. Y para ello hay que proporcionarle espacios de participación: la mujer embarazada y víctima de VG debe poder tener la oportunidad de explicar la situación que está viviendo y de obtener 
ayuda, al tiempo que debe poder expresar sus preferencias en relación con su embarazo y parto.

La participación es importante como estrategia, porque las capacidades se adquieren ejerciéndolas. Solo con espacios de participación la mujer desarrollará sus capacidades, recuperando las que la violencia le hubiera coartado y, en último término, recuperando su autonomía (como capacidad efectiva de tomar decisiones, más allá del derecho, que ya lo tenía).

Cuanto más autónomo uno es, más confianza y seguridad tiene para participar, más capacidades desarrolla y más cuotas de autonomía alcanza. Es un círculo virtuoso. Analicemos más en detalle todos estos conceptos: vulnerabilidad, autonomía, desarrollo de capacidades y participación.

\subsection{Vulnerabilidad}

Etimológicamente, el término latín vulnus significa herida. La vulnerabilidad es la capacidad de ser herido o la falta de recursos para rehacerse de una herida. Podemos establecer capas de vulnerabilidad (Luna, 2008):

La vulnerabilidad es una condición universal, una característica intrínseca a todo ser humano, en la medida en que todos podemos ser heridos física, moral o socialmente, porque vivimos inmersos en una red de relaciones de la que dependemos. Se la ha llamado vulnerabilidad antropológica (Delgado, 2016) o precariousness (Butler, 2004).

Pero, además, la vulnerabilidad es una experiencia particular: hay personas más vulnerables que otras. En este sentido, podemos establecer otras dos capas.

Por un lado, la vulnerabilidad como situación, que es temporal. La persona ha sido o puede ser herida y necesita ayuda del entorno para reponerse o para evitar la herida. Por ejemplo, la aparición de una enfermedad leve supone una situación temporal de vulnerabilidad, en la que la persona necesita la ayuda de los médicos para recuperarse. Las mujeres embarazadas están «en situación de vulnerabilidad» y necesitan la ayuda de las matronas para tener un buen proceso de gestación y parto.

La última capa es la vulnerabilidad como susceptibilidad, que es permanente. La persona tiene más posibilidades de ser herida y, si lo es, tiene más posibilidades de salir maltrecha o de no salir adelante (Morondo, 2016). En una intensidad alta, se la llama vulnerabilidad sociopolítica (Delgado, 2016), precarity (Butler, 2004) o indefensión 
(Morondo, 2016). Está causada por campos de poder que crean grupos sociales con menos cantidad y calidad de recursos y de oportunidades. En consecuencia, estos grupos sociales deben reclamar reconocimiento para autoconstruirse. Las mujeres, tradicionalmente, hemos tenido menos poder que los hombres y hemos tenido que reclamar reconocimiento para construir nuestra identidad más allá de los roles de madre y de ama de casa. Esta capa de vulnerabilidad puede dar lugar a nuevas vulnerabilidades temporales.

Las mujeres no embarazadas tienen dos capas, la condición universal (son seres humanos) y la situación permanente (son mujeres). Por eso el embarazo es un momento de especial vulnerabilidad, porque implica añadir la tercera capa, la situación temporal. Además, embarazadas o no, todas las mujeres son susceptibles de sufrir VG, puesto que no hay un perfil único de víctima (tienen otra vulnerabilidad permanente, además de la de ser mujer). Finalmente, la mujer embarazada y víctima de VG, tiene las tres capas: condición universal (ser humano), la situación permanente (mujer) y la situación temporal, que es doble (embarazo y contexto de violencia). La vulnerabilidad temporal generada por el contexto de violencia puede devenir permanente si la mujer no tiene herramientas para revertir la situación y además puede generar nuevas vulnerabilidades temporales, como problemas de salud derivados de las heridas o, incluso, la pérdida del hijo (aborto).

Estas tres capas de vulnerabilidad tienen en común que lo que resulta herido son los intereses de bienestar (well-being) y de autonomía (agency) (Martin, Tavaglione, y Hurst, 2014). ${ }^{1}$ La vulnerabilidad puede aumentar la dependencia de la persona que tiene que ayudar a la mujer a hacer frente a la herida. En situación de VG, la vulnerabilidad genera una dependencia especial, porque quien tendría que dar seguridad física, moral y social a la mujer (su pareja) se convierte en su agresor; y este, además, la coacciona e inhibe hasta tal punto que impide que pueda pedir ayuda a otras personas de su entorno. No obstante, la vulnerabilidad tiene una capacidad generativa: no debe verse como una debilidad, sino como un punto de partida para forjar la autonomía. La matrona debe ver la vulnerabilidad de la mujer como la oportunidad para que sea consciente de las capacidades que tiene (por pocas que sean, todos tenemos capacidades) y para ayudarla a fortalecerlas a fin de que gane autonomía y pueda gestionar la situación de violencia.

\footnotetext{
${ }^{1}$ Los autores usan el término «agencia» pero lo definen de forma confusa, por lo que en este artículo se usará el término «autonomía».
} 


\subsection{Autonomía}

La adquisición de autonomía es un proceso gradual, inversamente proporcional al de vulnerabilidad. Pero como la vulnerabilidad es una condición universal del ser humano, nunca habrá una autonomía absoluta; y como la autonomía es un derecho de la persona, además de una capacidad, nunca habrá una vulnerabilidad total. En este sentido, la matrona debe legitimar el sentimiento de vulnerabilidad (miedo, indefensión) de la mujer embarazada y víctima de VG, puesto que todo ser humano es vulnerable y ha sentido miedo e indefensión alguna vez; y también debe transmitirle que mantiene y mantendrá siempre parte de su autonomía, entendida como su derecho a tomar decisiones.

La autonomía tiene dos dimensiones: moral y funcional. La capacidad de tomar decisiones (autonomía moral) no puede desligarse de la capacidad de ser autosuficiente en la vida cotidiana (autonomía funcional): las decisiones deben poder hacerse efectivas y mantenerse en el tiempo. Tomar la decisión de separarse del marido no sirve de mucho si no puede llevarse a la práctica (si no se tiene casa, trabajo, capacidad de planificación del día a día, apoyo en el cuidado del bebé, relaciones fortalecidas para evitar nuevas dependencias...). Igualmente, tampoco sirve de mucho evitar la coacción del marido en la consulta si, después, en casa, ella no puede llevar a la práctica las decisiones o acuerdos tomados en relación al cuidado de su embarazo y de su bebé.

La autonomía es relacional. Las decisiones que tomamos afectan, positiva o negativamente, a los demás, quienes a su vez facilitan o dificultan nuestras decisiones. Las decisiones del agresor (ej. agredir, aislar) pueden afectar negativamente a la mujer, porque pueden afectar a su salud física, psíquica y social. Por otra parte, tomar decisiones depende tanto de la razón como de las emociones y de los sentimientos ligados a las decisiones que debemos tomar (Busquets, 2016), lo que implica que la matrona debe ayudar a la mujer a fortalecer su capacidad de razonamiento, y a detectar y a gestionar sus emociones. Es importante que sea la mujer quien decida en todo momento sobre su embarazo y parto, ya que la situación que se genera cuando las decisiones las toma la pareja, aunque haya un aparente clima de diálogo y consenso, se puede considerar una situación de violencia. En cambio, cuando la matrona ayuda a la mujer a tomar las decisiones por sí misma, la está ayudando a reconstruir su red de apoyo social, de la que la misma matrona forma parte. Está fortaleciendo la autonomía relacional de la mujer. 


\subsection{Desarrollo de capacidades}

El profesional no empodera, no crea capacidades en el paciente, sino que es el paciente quien se empodera a sí mismo, desarrollando capacidades, con la ayuda del profesional.

La filósofa Martha C. Nussbaum (2007) define las capacidades como áreas de libertad que las personas deben tener y poder ejercer para vivir una vida digna. Dependen de las habilidades internas de cada persona, por lo que son aquello que las personas son capaces de ser y hacer. Pero también dependen de las oportunidades ofrecidas por el entorno (político, social y económico), de modo que son condiciones, derechos sociales innegociables. En el caso de las mujeres embarazadas y víctimas de VG, las matronas son personas de su entorno que pueden generarles oportunidades.

La dignidad tiene que ver con la calidad de vida, que tiene tres dimensiones: objetiva (lo que dice la historia clínica), subjetiva (el grado de satisfacción vital) y relacional (la calidad de la red de apoyo social). Los psicólogos Schalock y Verdugo (2007) proponen ocho características de la calidad de vida. Dos de ellas, el desarrollo personal y la autodeterminación, son transversales a las tres anteriores dimensiones, puesto que el desarrollo de la persona debe ser físico, psíquico y social, y la toma de decisiones (autodeterminación) también debe poder darse en estas tres áreas. Las otras seis características que Schalock y Verdugo atribuyen a la calidad de vida podrían considerarse también transversales, porque al fin y al cabo todo aspecto de la vida de una persona influye en los demás, pero aún y así entendemos que afectan más directamente a una dimensión que a las otras. Las clasificamos así:

- Objetiva

- Bienestar físico

- Bienestar material

- Subjetiva

- Bienestar emocional

- Relacional

- Relaciones interpersonales

- Inclusión social

- Derechos

Para Nussbaum, la calidad de vida se mide en términos del grado de desarrollo de capacidades para la vida digna. Propone una lista de diez capacidades básicas que hay 
que garantizar, aunque, después, la persona no las utilice. A continuación, se muestran las definiciones de Nussbaum sintetizadas (entre comillas) y su adaptación a la mujer embarazada y víctima de VG. En algún caso, la relación de la definición de Nussbaum con la VG es tan evidente que no se ha añadido ninguna explicación adicional:

1. Vida: «ser capaz de vivir una vida humana de longitud normal hasta el final. No morir prematuramente». Implica que la mujer no muera por homicidio o suicidio.

2. Salud corporal: «ser capaz de tener buena salud, incluyendo la salud reproductiva». Implica que la mujer pueda tomar las decisiones en relación con el embarazo y parto, en vez de que las tome la pareja.

3. Integridad corporal: «ser capaz de moverse con libertad; tener seguridad ante ataques violentos, incluyendo la agresión sexual y la violencia doméstica; tener oportunidades para la satisfacción sexual y para elegir en materia de reproducción».

4. Sentidos, imaginación y pensamiento: «ser capaz de utilizar los sentidos, de imaginar, de pensar y de razonar [...] mediante una educación adecuada; ser capaz de usar la libertad de expresión; y ser capaz de tener experiencias de placer y de evitar el dolor no beneficioso». Implica que la mujer tenga formación, por ejemplo, sobre sus derechos; y en concreto, sobre sus derechos en materia de reproducción y salud. También implica que pueda expresar sus opiniones libremente, y que tenga una sexualidad sana, consentida y libre, en vez de coaccionada y basada en la agresión.

Emociones: «no ver el propio desarrollo emocional deteriorado por el miedo y la ansiedad». Implica que la mujer sea capaz de identificar, expresar y gestionar sus emociones.

5. Razonamiento práctico: «ser capaz de formular una concepción del bien y de hacer una reflexión crítica sobre la planificación de la propia vida». Implica que la mujer sea capaz de formular que la agresión (física o psicológica) no es justificable, ni culpa suya, y que sea capaz de planificar de forma realista cómo revertir la situación.

6. Afiliación: «a) ser capaz de interacción social y empatía; b) tener las bases sociales de autorrespeto y no humillación para ser tratado como un ser con dignidad y con igual valía que los demás». Implica que la mujer sea capaz de establecer vínculos con otras personas, fuera de su relación de pareja, para no 
asilarse, así como que experimente relaciones de reconocimiento mutuo que le permitan ver que su pareja no la trata como un ser con dignidad.

7. Otras especies: «cuidar de los animales, las plantas y la naturaleza». Aunque no parezca aplicable, es relevante el hecho de que muchas víctimas no quieren separarse porque las casas de acogida no admiten mascotas.

8. Juego: «ser capaz de reír y de disfrutar de actividades recreativas». La alegría es una de las cuatro emociones básicas, junto con la tristeza, el miedo y la rabia (Antoni y Zentner, 2014). Además, el ocio es saludable, en tanto que fomenta el bienestar físico, mental y social (ver Benkö, Modi y Tarkó, 2017), lo que concuerda con las tres dimensiones de la calidad de vida.

9. Control sobre el entorno: «a) político: poder gobernar la propia vida y tener oportunidades de participación política [...]; b) material: tener propiedades y un trabajo». En cuanto al entorno político, implica que la mujer sea capaz de mantener o recuperar su proyecto vital a pesar del contexto de violencia, a lo que la puede ayudar la participación en ámbitos comunitarios (asociaciones, entidades, etc.). En relación con el entorno material, implica que la mujer pueda trabajar y preservar propiedades para sí, fuera del control de la pareja, como la casa o el coche. Ambos sentidos, político y material, tienen que ver con el hecho de que la mujer preserve su autonomía funcional para poder hacer efectivas sus decisiones y mantenerlas en el tiempo.

\subsection{Participación}

Hemos dicho que fortalecer capacidades requiere oportunidades de participación para ejercerlas.

Si entendiéramos la vulnerabilidad como condición universal, habría una igualdad formal: necesitamos igualdad de oportunidades para satisfacer nuestros intereses de bienestar y autonomía. Pero si entendemos la vulnerabilidad como una experiencia particular, hay una desigualdad inherente: hay personas más vulnerables que otras, que necesitan más oportunidades, porque tienen menos recursos o menos autonomía para transformarlos en bienestar.

La importancia de la participación se deduce del planteamiento de Amartya Sen (Drèze y Sen, 1997): el grado de desarrollo de una comunidad no depende de la cantidad de recursos que tenga, sino de las capacidades de sus miembros para transformarlos en 
bienestar. Por lo tanto, la manera de ayudar a las comunidades en desarrollo no es proporcionarles más recursos, sino fortalecer las capacidades de sus miembros. Como se dice popularmente, "no se trata de ofrecerles comida, sino de enseñarles a pescar y cultivar". El filósofo Ángel Puyol (1999) lo extrapola al ámbito sanitario: los pacientes necesitan oportunidades para ejercer sus capacidades y, así, transformar la asistencia médica que reciben en salud efectiva (calidad de vida en sus tres dimensiones). ${ }^{2}$

\section{ACOMPAÑAMIENTO COMO PROCESO DE CONSTRUCCIÓN DE UN ENTORNO DE CONFIANZA Y RESPETO EN LA CONSULTA}

Hasta ahora hemos hablado del acompañamiento biopsicosocial como proceso mediante el cual la matrona ayuda a la mujer embarazada y víctima de VG a empoderarse a sí misma, fortaleciendo sus capacidades de autocuidado y de cuidado de su bebé; así como sus capacidades para gestionar la situación de violencia y recuperar su proyecto vital.

Este proceso de empoderamiento requiere que la consulta sea un entorno en el que se pueda construir un vínculo de confianza y respeto entre la matrona y la mujer, que permita que la mujer se sienta segura para expresar sus sentimientos, miedos o dudas. La creación de este vínculo es necesario para cualquier mujer embarazada, pero aún lo es más si la mujer está sufriendo violencia, porque se añade, a los miedos y dudas sobre el embarazo, y la crianza, el miedo, la incertidumbre y la impotencia generadas por la situación de violencia. Este vínculo de confianza y respeto tiene tres pilares (fig. 2). El objetivo del vínculo es proteger a la mujer, en la medida de lo posible, ofreciéndole recursos para poder salir de la situación de violencia. Es importante que, en la consulta, se sienta protegida; de lo contrario, si se siente cuestionada en sus decisiones, es probable que no vuelva y se pierda la continuidad asistencial.

El modo de hacer sentir a la mujer protegida es tener un diálogo sincero y efectivo con ella que le permita expresarse y que ayude a la matrona entender su situación y sus decisiones. Para ello, es necesario reconocer a la mujer como una persona vulnerable, pero con dignidad y con capacidad para empoderarse a sí misma y revertir su situación.

${ }^{2}$ El modelo salutogénico del sociólogo Aaron Antonovsky (1996) se basa las mismas ideas: las personas y la comunidad deben potenciar sus propios recursos, entre los cuales están las capacidades, que son activos de salud. 


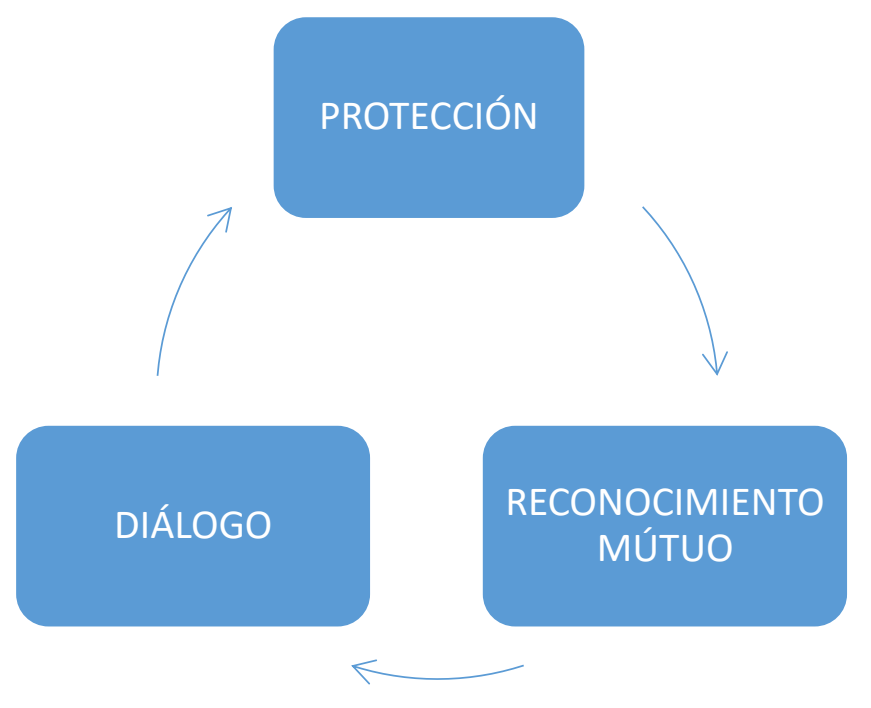

Figura 2. Modelo de acompañamiento como proceso de construcción de en un entorno de confianza en la consulta (elaboración propia)

Volvemos a tener un círculo virtuoso: cuanto más protegidas se sientan las mujeres, más reconocidas se sentirán y más fácil será que se abran y hablen con la matrona. Y cuanto más se abran y hablen, más fácil será para la matrona entenderlas y protegerlas y, por lo tanto, más les estará reconociendo y facilitando el diálogo.

Analicemos todos estos elementos: protección, reconocimiento y diálogo. En el análisis se verá que estos elementos tienen que ver con los cuatro principios de ética biomédica (Beauchamp y Childress, 1989) y los cuatro modelos de relación asistencial (Emmanuel y Emmanuel, 1992).

\subsection{Protección}

Uno de los cuatro principios de ética biomédica, y al que más importancia se le otorga, es el principio de autonomía, que tiene dos partes: por un lado, respetar las decisiones de los pacientes competentes; y por el otro, proteger los pacientes con autonomía reducida.

En el caso de las mujeres embarazadas y víctimas de VG están en juego ambas partes. La matrona debe respetar las decisiones de la mujer, en la medida de lo posible, para que ella sienta que tiene el control sobre su proceso de embarazo y parto; pero ello no significa que deba respetarlas siempre, ya que la situación de violencia y dependencia que vive la mujer puede conllevar a que no sea competente para tomar ciertas decisiones. En este caso, la matrona deberá protegerla, procurando que no tome decisiones potencialmente 
perjudiciales para sí misma (ej. decirle a su pareja que quiere separarse cuando todavía está preparando el plan de fuga). La matrona debe encontrar el equilibrio entre respetar las decisiones de la mujer y protegerla; de lo contrario, si la sobreprotege, imponiendo el criterio profesional, ya sea en relación con la situación de violencia o con el proceso de embarazo y parto, podría llegar a reproducirse el modelo impositivo, autoritario, del agresor (Gómez et al., 2017).

Este equilibrio requiere evitar dos extremos, dos modelos de relación asistencial perjudiciales: el paternalista (imponer el criterio profesional) y el informativo (limitarse a transmitir información sobre los recursos disponibles).

Para evitar el paternalismo, hay que ejercer bien el principio de beneficencia. Este principio afirma que hay que hacer el bien teniendo en cuenta lo que el paciente considere bueno para él. Las mujeres víctimas de VG pueden tener alterado el concepto de bien, de lo que es mejor para ellas, por ejemplo, si justifican o no reconocen la gravedad de las agresiones o si viven en un marco cultural que entiende que es bueno imponer disciplina a la mujer. O puede que sí sean conscientes de que lo mejor para ellas es separarse, pero que no sean capaces de dar este paso por miedo o por falta de autonomía funcional.

Por otro lado, para evitar una relación meramente informativa, habrá que ejercer bien el principio de justicia. Este principio defiende la distribución equitativa de los recursos disponibles. Equitativa no quiere decir igualitaria: no se trata de dar lo mismo a todos, sino a cada uno según sus necesidades. Ello significa que, aunque es necesario que las matronas tengan información sobre todos los recursos disponibles, no es recomendable dársela por igual a todas las mujeres, ni toda de golpe. Hay que atender a la situación de violencia específica de cada mujer para ofrecerle aquellos recursos que necesite, a medida que los necesite.

\subsection{Diálogo}

Un diálogo sincero y efectivo requiere habilidades comunicativas (empatía, asertividad, lenguaje no verbal, calidez, mirada y escucha atentas...). Estas habilidades son necesarias para forjar una relación asistencial positiva, una mezcla entre las relaciones interpretativa y deliberativa.

La relación interpretativa se basa en ayudar a la persona, a la mujer, a descubrir sus valores, insertos en su narración vital, para ver cuál de las alternativas terapéuticas o personales los respeta más. 
La relación deliberativa se basa en ayudar a la persona, a la mujer, a reflexionar sobre los valores relacionados con la salud para ver cómo las distintas alternativas van a afectar a la suya.

Una relación asistencial interpretativa y deliberativa, en la que los profesionales no caigan en el error de imponer sus valores o su concepción de la salud, posibilitará la toma de decisiones acompañada y la responsabilidad compartida: la matrona tiene la responsabilidad de respetar y proteger a la mujer, y la mujer necesita encontrar el modo de superar la dependencia respecto del agresor para poder volver a responsabilizarse de su vida.

\subsection{Reconocimiento}

Uno de los requisitos de la ética dialógica (Habermas, 2010; Apel, 2005) es el reconocimiento mutuo de todos los participantes en el diálogo.

Axel Honneth (2009) sostiene que satisfacer las expectativas de reconocimiento transforma relaciones conflictivas en cooperativas. En cambio, lesionarlas origina relaciones conflictivas, porque obliga a una lucha por el reconocimiento. Este filósofo propone tres esferas de reconocimiento:

- Emocional: reconocimiento de la persona como sujeto de necesidades, lo que le genera autoconfianza.

- Legal: reconocimiento de la persona como sujeto de derechos y deberes, lo que le genera autorrespeto.

- Social: reconocimiento de la persona como sujeto de capacidades, lo que le genera autoestima.

La matrona debe reconocer a la mujer víctima de VG en las tres esferas para que ella reconozca a la matrona como persona de confianza que la puede y quiere ayudar. Solo así forjarán una relación de cooperación que proporcione herramientas a la mujer para resolver su situación.

\section{RELACIONES INTERPROFESIONALES}

Las matronas no trabajan solas: pertenecen a equipos donde hay otros profesionales, forman futuras matronas, deben coordinarse con otros centros (ej.: entre hospital y 
Atención Primaria), deben responder ante dirección, y su trabajo se puede ver afectado por decisiones de gerencia (ej.: decisiones presupuestarias). Si las relaciones interprofesionales no funcionan bien, las matronas difícilmente se sentirán a gusto haciendo su trabajo. De hecho, muchas refieren cierto intrusismo profesional por parte de ginecólogos y obstetras. Pero, además, se pueden producir situaciones en las que el propio ámbito sanitario sea generador de violencia hacia las mujeres (violencia obstétrica).

La presencia de violencia obstétrica vulnera el cuarto principio de ética biomédica, la no-maleficencia, que dice que hay que evitar daños innecesarios a los pacientes. Además, pone en riesgo la confianza de la mujer en la matrona, lo que puede dificultar el desempeño de los dos círculos virtuosos anteriores. La filósofa Annette Baier (1995) define la confianza como el hecho de aceptar la vulnerabilidad que implica que otros puedan causar un daño a aquello que uno considera valioso - el cuerpo, el bebé- porque se juzga que no lo causarán.

Es necesario establecer un tercer círculo virtuoso (fig. 3).

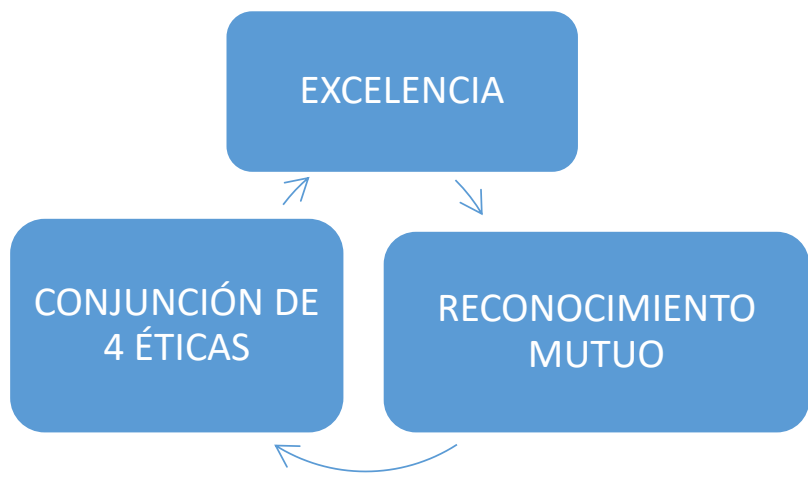

Figura 3. Modelo de gestión de la calidad asistencial (elaboración propia)

Como cualquier profesional, el objetivo último de la matrona es conseguir la excelencia, es decir, ofrecer un servicio (asistencia sanitaria) de la máxima calidad posible, entendiendo la calidad en su dimensión técnica, pero también ética.

El modo de conseguir la excelencia es encontrar el encaje de cuatro éticas: personal, cívica (social), profesional (código deontológico) y de la organización. Dicho encaje requiere establecer relaciones de reconocimiento mutuo: los profesionales deben reconocerse entre ellos en sus respectivos roles, tanto en sentido vertical (entre jefes y trabajadores) como en sentido horizontal (entre compañeros).

He aquí el tercer círculo virtuoso: reconocer el rol de los otros, y ser reconocido por los demás en el propio rol, favorece el encaje de las cuatro éticas en juego, lo que facilita 
la excelencia profesional. Y al mismo tiempo, cuanto más excelente uno es, más satisfacción tiene con el propio trabajo (individual y de equipo), lo que ayuda a mantener el reconocimiento de los diferentes roles y el encaje de las cuatro éticas, aumentándose así la excelencia.

\section{REFLEXIÓN FINAL}

El modelo de acompañamiento biopsicosocial propuesto no debe aplicarse solamente en situaciones complejas, como la de una mujer embarazada víctima de VG, sino que debería fundamentar la actitud de cualquier profesional sanitario hacia cualquier paciente, porque, como dice la enfermera Myra Levine (1971), la conducta ética no es la rectitud moral en tiempos de crisis, sino el compromiso con los demás en las interacciones diarias. Los principios éticos deberían regir no solo la gestión de las situaciones de crisis, sino todas las relaciones humanas, en tanto que relaciones entre seres vulnerables, y entre las que se incluyen las relaciones profesionales.

\section{AGRADECIMIENTOS}

A la Dra. Josefina Goberna y al proyecto 016ACUP 00083 «Prevenció i Detecció de les Violències de Gènere durant l'embaràs: de la violència masclista a la violència obstètrica», en el cual se inscribe el presente artículo, por permitirme integrarme en la formación dirigida a matronas la cual fue el origen del presente artículo. 


\section{BIBLOGRAFÍA}

ANTONI, M.; J. ZenTneR. Las Cuatro Emociones Básicas. Barcelona: Herder, 2014.

Antonovsky, A. The salutogenic model as a theory to guide health promotion. En: Health Promotion International. 1996, vol. 11, pp. 11-18.

APEL, K.O. Ética del discurso y ética de la liberación. Madrid: Trotta, 2005.

BAIER, A. Moral Prejudices. Harvard: Harvard University Press, 1995.

Beauchamp, T. L.; J. F. ChILDRESS. Principles of biomedical ethics. New York: Oxford University Press, 1989.

Benkö, Z.; I. Modi; K. TARKÓ (eds.). Leisure, Health and Wellbeing. A Holistic Approach. London: Palgrave Macmillan, 2017.

Busquets, M. La autonomía relacional. En: Boladeras, M.; J. Goberna (eds.). Bioética de la maternidad. Humanización, comunicación y entorno sanitario. Barcelona: Edicions Universitat de Barcelona, 2016, pp. 83-90.

BUTLER, J. Precarious life: The powers of mourning and violence. London: Verso, 2004. DELGADO, J. Vulnerabilidad y fragilidad. La importancia de los vínculos. En: Boladeras, M.; J. Goberna (eds.). Bioética de la maternidad. Humanización, comunicación y entorno sanitario. Barcelona: Edicions Universitat de Barcelona, 2016, pp. 91-100.

DrÈZE, J.; A. SEN. India: Development and Participation. Nueva York y Oxford: Oxford University Press, 1997.

EMANUEL, E. J.; L. L. EMANUEL. Four models of the physician-patient relationship. En: JAMA. 1992, vol. 267, núm. 16, pp. 2221-2226.

EstÉVEZ, E. P. Intervenciones de enfermería en la atención a las mujeres víctimas de violencia de género en las consultas de Atención Primaria de salud. En: MUSAS. 2017, vol. 2, núm. 2, pp. 75-01.

GARCÍA-MARZÁ, D. Ética empresarial. Del diálogo a la confianza. Madrid: Trotta, 2004.

GómEZ-FERnÁNDEZ, A., et al. Aspectos bioéticos de la relación asistencial de las matronas con las mujeres embarazadas en contexto de violencia de género. En: MUSAS. 2017, vol. 2, núm. 2, pp. 40-55.

HABERMAS, J. Teoría de la acción comunicativa. Madrid: Trotta, 2010.

HONNETH, A. Reconeixement i menyspreu: sobre la fonamentació normativa d'una teoria social. Barcelona: Centre de Cultura Contemporània de Barcelona, 2009.

LEVINE, M. E. Holistic nursing. En: The Nursing Clinics of North America. 1971, vol. 6, núm. 2, pp. 253-264. 
LuNA, F. Vulnerabilidad: la metáfora de las capas. En: Jurisprudencia Argentina. 2008, vol. 4, núm. 1, pp. 60-67.

Martin, A. K.; N. Tavaglione; S. Hurst. Resolving the conflict: clarifying «vulnerability» in health care ethics. En: Kennedy Inst Ethics J. 2014, vol. 24, núm. 1, pp. 51-72.

MORONDO, D. ¿Un nuevo paradigma para la igualdad? La vulnerabilidad entre condición humana y situación de indefensión. En: CEFD. 2016, vol. 34, pp. 205-221.

Nussbaum, M. C. Las fronteras de la justicia: consideraciones sobre la exclusión. Barcelona: Paidós, 2007.

PUYOL, A. Justícia i Salut. Ètica per al racionament dels recursos sanitaris. Barcelona: Edicions Universitat Autònoma de Barcelona, 1999.

SCHALOCK, R. L.; M. A. VerdugO. El concepto de calidad de vida en los servicios y apoyos para personas con discapacidad intelectual. En: Revista Española sobre Discapacidad Intelectual. 2007, vol. 38, núm. 224, pp. 21-36

VELASCO, C. Recomendaciones para la detección precoz de la violencia en el embarazo. En: Matronas Profesión. 2008, vol. 9, núm. 3, pp. 32-37. 